\title{
The Challenge of Quality Improvement at the System Level. Whither CCO?
}

Adalsteinn Brown

\begin{abstract}
Cancer Care Ontario has made significant strides in the improvement of access to high-quality cancer care by putting in place the basic elements of transformation: a clear plan, reporting against this plan and clinician engagement. However, as our health system changes so must improvement efforts. Greater focus on equity and population health will require a better understanding of the people served. The increasing need for improvement will put a premium on quality improvement skills. Finally, the need to re-engineer care across the patient journey will require attention to integration; attention that will have to come from government.
\end{abstract}

\section{Background}

In the decade since the release of the first cancer plan, Cancer Care Ontario (CCO) has made a number of efforts to improve the quality of cancer care and to build a platform for cancer control. These efforts are well-documented in other papers and include the creation of the Cancer Quality Council of Ontario (Sullivan et al. 2004), the creation of the first strategy-based cancer system scorecard (Greenberg et al. 2005) and its subsequent evolution into the Cancer System Quality Index (CSQI), the development of new programs designed to increase the effectiveness and appropriateness of care such as the deployment of synoptic pathology and the introduction of the first integrated cancer screening program in Canada to meet the criteria for organized screening programs. All of these efforts build upon earlier work that laid a strong foundation for evidence-based care such as the Program in Evidence-Based Care (Browman 2012) and efforts to repatriate and increase access in Ontario to needed cancer care. In recognition of its success, CCO may point to its status as the longest-running independent health agency in the province and a growing list of responsibilities given to it by the government such as the Access to Care program, for all surgeries that was critical to the success of the Wait Times Strategy in Ontario, and the Ontario Renal Network that has brought about rapid and important improvements in the quality of dialysis care. Perhaps as importantly, CCO has published extensively on these efforts and consistently reported performance data, even when it showed poor results.

\section{Has Cancer Care Quality Improved?}

In order to argue whether quality has improved, we should first define what we mean by quality. Although organizations like the Institute of Medicine have defined key dimensions of quality and the Excellent Care for All Act (2010) enshrines an even longer list in Ontario, our notion of what constitutes quality care has evolved over time. In the first several years of this century, quality of care was arguably focused on more and faster care. Following on the heels of substantial reductions in capacity and 
streamlining, policy efforts at the federal and provincial level were focused on reducing wait times and increasing resources (facilities and professionals) available to provide care. Within this relatively narrow definition of quality of care, the quality of cancer care has improved. Wait times for cancer surgery have declined - and declined by more than some of the other priority areas identified by the federal and provincial governments in 2004 - and several new regional cancer centres have opened, while the capacity for radiation treatment has nearly doubled and improved in quality. Reports of cancer patients seeking care outside of Ontario seem to be less prominent, and most debates around access deal with new treatments for which evidence is inconsistent.

Although more and faster care was clearly the policy priority at the beginning of this century, the focus of efforts to improve quality has since expanded to include the technical quality (appropriateness and effectiveness) of care and, to a lesser degree, the extent to which this care is patient-centred. Once again, quality seems to be improving, although the evidence is less clear. Big dot indicators like hospital-specific mortality rates are declining in Ontario, and key cancer indicators related to the quality of care provided such as survival are at international benchmarks. Based on the CONCORD study that aggregates population-based cancer survival data from across dozens of jurisdictions, one- and five-year survival for the four most common cancers is as good in Ontario as in any other jurisdiction (Coleman et al. 2011). This performance is reflected in largely positive ratings on care in the CSQI (2014). It is also linked to major initiatives around measuring and improving the quality of surgical care, the appropriateness of radiation and chemotherapy and a continuing leadership role for clinicians in generating and adopting evidence on effective care. $\mathrm{CCO}$ has also led in this regard by implementing symptom assessment efforts that have improved the quality of life and patient experience measurement at all regional cancer centres.

More recently, however, the focus of quality improvement efforts has expanded to include broader goals. These have been variously defined as the Triple Aim (population health, patient experience and cost), value (a ratio of quality and cost) and even equity (reducible harm is eliminated across all populations and care is sensitive to the community). These perspectives on quality are captured in the CSQI. But we could argue that the current scope of measurement does not match the scope of these goals and the results, where they are measured, include some of the poorest ratings.

$\mathrm{CCO}$ is not alone in struggling to meet the challenges posed by these broader goals. It is clear that no jurisdiction or system has successfully met all of these goals. Major new policy initiatives around the world like Accountable Care Organizations in the United States or Health Links in Ontario are intended to address some of these broader goals, but there is no silver bullet or even clear and compelling evidence on how to deal successfully with all of these challenges simultaneously. As CCO moves forward, it may be useful to consider what has helped drive its success through the first two evolutions of quality (more care and higher-quality care) and whether these lessons can shape CCO's and, more broadly, all health systems' strategies for improvement going forward.

\section{Stay the Course?}

As always, the credit for past success goes to the people working in the cancer system at every level. Improvement happens because they do things differently, not because of policy or programs. But it may be useful to consider some of the important enablers that CCO has put in place that encourage and enable improvement. CCO has implemented a wealth of new programming, introduced new information systems and created new roles and goals. Perhaps most notably, CCO has also evaluated many of these innovations, keeping those that work while modifying some of the others. More fundamentally, CCO has also implemented what we have called the table stakes of transformation. In an earlier piece in this journal we suggested that successful health systems have gotten three things right: they have created a plan with clear goals, reported against those goals in a way that people had to pay attention and they have engaged clinicians in improvement efforts against those goals (Brown et al. 2012). CCO has met these tests.

Since the first cancer plan in 2004, CCO has regularly updated the cancer plan and set both programmatic and outcome goals every year. This document is publicly available and widely distributed across the cancer system. Likewise, the first comprehensive cancer scorecard came out publicly in 2005 and has been updated every year, evolving over time to reflect many aspects of quality and the cancer patient journey. Clinical leadership and engagement in improvement efforts at every level from the board table to the bedside has continued to be a hallmark of CCO's efforts. These are critical elements to ensure quality, but will they be enough as the goals for improvement change?

One of the first things to consider is whether the health system has changed substantially enough that these elements should also change. The short answer is no. It is true that we have many more agencies working in the Ontario health system and many more sets of publicly reported indicators. But none of these changes would argue for more than a need for greater collaboration. Another issue worth consideration is whether we now know more about quality and quality improvement that invalidates what we have called above the table stakes. The short answer again is no. There has been excellent work over the past decade on key aspects of health system transformation 
(Ham 2010; Baker et al. 2008). But again, none of these insights argue against the importance of a plan, reporting and clinical leadership. Rather, they provide additional and more refined advice on how to improve quality across health systems in a sustainable way that complements and reinforces the importance of plans, reporting and clinical leadership.

Finally, we must consider whether the goals of quality improvement have changed so much that the historic approach is no longer sufficient. Here, the answer is yes. The new goals require new measures and, perhaps more importantly, the acceptance of these measures as a point of meaningful accountability for managers and providers in the cancer system and, more broadly, the health system. If we want to pursue equity seriously in the health system, we need to know more about the people who need care and what they need. In contrast to the United States, we do not systematically collect information on the background of people seen in our system and we rely on ecological extrapolations from census data to know whether access is as good for the poor as it is for the wealthy. We know little about whether people understand the instructions for care given to them. Likewise, our efforts to engage patients meaningfully in defining what they need from care remain in their infancy. Moving beyond equity to a question of simple effectiveness, we should not underestimate the pace of technological change in care and the degree to which issues like personalized or precision medicine will fundamentally change what data we collect and what data we use to make decisions and even how we make these decisions at the bedside or in the boardroom. In this regard, $\mathrm{CCO}$ and cancer agencies in general can lead health systems as they did with wait times. Building off of its current information management policies, $\mathrm{CCO}$ can continue to stretch what data are collected and how they are used to make better decisions.

But more and better data will not be sufficient to meet the new goals of the quality movement. Success will also require increased leadership and improvement capacity. The broader goals require a level and breadth of improvement activity that is not yet common across most health systems. $\mathrm{CCO}$ has done an excellent job of engaging clinicians across the cancer system to shape and lead policy implementation. What is still missing - as it is in most systems - is the ability to engage a large portion of the professionals working in the system in improvement exercises. This is the oft-repeated mantra of Toyota and other organizations that everyone's job is to meet a standard and raise that standard. This vision of our system will require increased training in improvement as well as increased recognition that quality improvement is a portion of people's work that must be included when determining workload and compensation. CCO can lead on this goal but it will likely need to lead in partnership, as the capacity to train professionals in quality improvement rests elsewhere in the system. In this situation, CCO can lead the entire system by increasing its focus on improvement and its recognition of the importance of improvement at all levels of the cancer system.

Finally, better data and more extensive improvement efforts will help achieve the current broader set of quality goals but two more transformations are critical. The first is a cultural shift. There is no single organization or individual who could credibly claim that they "have control over outcomes like cancer mortality or the equity of cancer mortality." Rather, many providers influence these goals. This limits the willingness of any actor in the health system to accept accountability for this sort of big dot indicator. But if $\mathrm{CCO}$ cannot translate this sort of indicator into something meaningful and acceptable to providers, and if it cannot include these indicators in its accountability agreements, it will not be able to pursue these indicators in a meaningful way. This will be a cultural undertaking of great significance but a worthwhile one where $\mathrm{CCO}$ can again lead the health system.

The second shift is a structural shift. The broader sets of goals like the Triple Aim look to improve both health and cost. There is an increasing weight of evidence that this sort of goal is possible but in order to realize these goals, there needs to be a way of connecting the costs and benefits of improvement efforts. Øvretveit (2009) has noted that the costs and benefits of improvement are spread out across time and across stakeholders. If we look to improve the accuracy of cancer diagnosis and support more effective surgical treatment, we merely need to focus on the hospital and can achieve huge gains through improved surgical pathology alone. If we want to increase the value of care, the connection across the cancer journey must be much tighter and we must be able to share costs and benefits across that chain. Initial work here has been excellent - for example, the work to increase links with primary care - but in a siloed system like Ontario's, it has likely gone as far as it can. This is where the argument for structural reform that has to come from a ministry is critical. CCO can support this argument but it has likely done as much as it can here.

Broader health system transformation is not where we want it to be. This is as true in Ontario as it is in any other jurisdiction in Canada. We lag other systems. That being said, we should not ignore our successes. The table stakes for transformation were put into place at $\mathrm{CCO}$ years ago. They have borne fruit. We falter in many international scorecards, but Canada (and Ontario) do very well in international comparisons of cancer survival; undoubtedly one of the best indicators of the quality of a cancer care system. The challenge now is to move from re-engineering a cancer care system to creating a cancer control system. CCO, as well as other groups like the Canadian Partnership Against Cancer, can lead the way towards this goal, sometimes through action, sometimes through advocacy and always by establishing the evidentiary basis. $\mathrm{HQ}$ 


\section{About the Author}

Adalsteinn Brown is a Director, Institute of Health Policy, Management and Evaluation and Dalla Lana Chair and Division Head, Dalla Lana School of Public Health, University of Toronto.

\section{Conflict of Interests}

Member of the Cancer Quality Council of Ontario since 2007; involved in the development of the first Cancer System Quality Scorecard for CCO (2004) and Indicators of Quality Cancer Surgery (2005). No compensation received for these efforts.

\section{Reference}

Baker, G.R., A. Macintosh-Murray, C. Porcellato, L. Dionne, K. Stelmacovich and K. Born. 2008. High-Performing Health Systems: Delivering Quality by Design. Toronto, ON: Longwoods Publishing.

Browman, G.P. 2012. "Challenges in Knowledge Translation: The Early Years of Cancer Care Ontario's Program in Evidence-Based Care." Current Oncology 19(1): 27-35.

Brown, A.D., G.R. Baker, T. Closson, T.S. Sullivan. 2012. "The Journey Towards High Performance and Excellent Quality." Healthcare Quarterly 15: s6-29.
Coleman, M.P., D. Forman, H. Bryant, J. Butler, B. Rachet, C. Maringe et al. 2011. "Cancer Survival in Australia, Canada, Denmark, Norway, Sweden, and the UK, 1995-2007 (the International Cancer Benchmarking Partnership): An Analysis of Population-Based Cancer Registry Data." Lancet 377(9760): 127-38.

Cancer Quality Council of Ontario. 2014. Cancer System Quality Index (CSQI) 2014. Retrieved June 24, 2014. <http://www.csqi.on.ca>.

Greenberg, A., H. Angus, T. Sullivan and A. Brown. 2005. "Development of a Set of Strategy-Based System-Level Cancer Care Performance Indicators in Ontario, Canada." International Journal for Quality in Health Care 17(2): 1078-114.

Ham, C. 2010. "The Ten Characteristics of the High Performing Chronic Care System." Health Economics, Policy, and Law 5(1): 71-90.

Øvretveit, J. 2009. Does Improving Quality Save Money. London: The Health Foundation.

Sullivan, T., M. Dobrow, L. Thompson and A. Hudson. 2004. "Reconstructing Cancer Services in Ontario." Healthcare Papers 5(1): 69-80. 